\title{
Results of Simple Conservative Treatment of Midfoot Charcot Arthropathy
}

\author{
You Keun Kim, $\mathrm{MD}^{*, \#}$, Ho Seong Lee, $\mathrm{MD}^{\dagger}$, Sang Gyo Seo, $\mathrm{MD}^{\dagger}$, Seung-Hwan Park, $\mathrm{MD}^{\dagger}$, \\ Dimas Boedijono, $\mathrm{MD}^{\ddagger}$ \\ ${ }^{*}$ Department of Orthopedic Surgery, Godoil Hospital, Seoul, \\ ${ }^{\dagger}$ Department of Orthopedic Surgery, Asan Medical Center, University of Ulsan College of Medicine, Seoul, Korea, \\ ${ }^{\ddagger}$ Department of Orthopedic and Traumatology, Foot and Ankle, Musculoskeletal Clinic, Fatmawati General Hospital, Jakarta, Indonesia
}

\begin{abstract}
Background: Traditionally, conservative management with an offloading orthosis, such as total contact cast (TCC), has been the standard of care for midfoot Charcot arthropathy. Considering complications of TCC and surgery, we treated midfoot Charcot arthropathy without TCC in our patients. The purpose of this study was to report clinical and radiological outcomes of conservative management of midfoot Charcot arthropathy.

Methods: A total of 34 patients (38 feet) who were diagnosed as having midfoot Charcot arthropathy between 2006 and 2014 were included. Patients started full weight bearing ambulation in a hard-soled shoe immediately after diagnosis. Outcomes such as progression of arch collapse, bony prominence, ulcer occurrence, limb amputation, and changes in Charcot stage were evaluated.

Results: Of 38 feet, arch collapse was observed in four while progression of bottom bump of the midfoot was observed in five feet. Foot ulcers related to bony bumps were found in two feet.

Conclusions: Conservative treatment without restriction of ambulation is recommended for midfoot Charcot arthropathy because it is rarely progressive, unlike hindfoot-ankle arthropathy. In some cases, simple bumpectomy can be required to prevent catastrophic infection.
\end{abstract}

Keywords: Charcot joint, Conservative treatment

Charcot arthropathy occurs in the presence of peripheral neuropathy due to diabetes mellitus (DM) or other neurologic disorders. Charcot arthropathy of the foot and ankle is a complicated sequela of DM, affecting $0.1 \%$ to $2.5 \%$ of diabetic patients. ${ }^{1)}$ However, the increasing age of the population has led to a rise in the prevalence of DM. Therefore, patients with Charcot arthropathy will also increase proportionally. ${ }^{2,3)}$ According to Frykberg et al., ${ }^{4)}$ among

\footnotetext{
${ }^{\#}$ Current affiliation: Department of Orthopedic Surgery, Bupyeong Himchan Hospital, Incheon, Korea

Received March 11, 2019; Accepted June 21, 2019

Correspondence to: Ho Seong Lee, MD

Department of Orthopedic Surgery, Asan Medical Center, 88 Olympic-ro 43-gil, Songpa-gu, Seoul 05505, Korea

Tel: +82-2-3010-3521, Fax: +82-2-488-7877

E-mail: hosng@amc.seoul.kr
}

patients with Charcot arthropathy, the most commonly involved joint is the tarsometatarsal joint (40\%), followed by naviculocuneiform, talonavicular, and calcaneocuboid joints (30\%), metatarsophalangeal and interphalangeal joints (15\%), and ankle and subtalar joints (10\%). Thus, the midfoot is the most common site of involvement in Charcot arthropathy. ${ }^{5,6)}$ Progressive collapse of the foot can lead to severe rocker-bottom deformity and recurrent plantar skin ulceration.

Charcot arthropathy of the hindfoot and ankle requires surgical stabilization because of the inability to successfully brace and stabilize these complex, multiplanar deformities. ${ }^{7)}$ However, the optimal management of midfoot Charcot arthropathy remains controversial. The goal of treatment, whether operative or nonoperative, is to achieve a plantigrade foot with osseous stability. Traditionally, conservative management with offloading devices 
Kim et al. Conservative Treatment of Midfoot Charcot Arthropathy

Clinics in Orthopedic Surgery • Vol. 11, No. 4, $2019 \bullet$ www.ecios.org

such as total contact cast (TCC) has been the standard of care. ${ }^{8}$ However, it is a time-consuming process with potential complications. ${ }^{9)}$ It is difficult to determine when and how long the TCC should be applied. In addition, in the presence of ulcer lesions, it is not easy to apply TCC because of the risk of wound aggravation.

Surgical treatment options for midfoot Charcot arthropathy range from simple bumpectomy to major reconstructive surgery. However, surgical reconstruction of midfoot Charcot arthropathy is difficult because of poor bone quality, bone loss, vasculopathy, and the presence of infection with or without soft-tissue loss. ${ }^{10,11)}$ And poor glucose control greatly increases the incidence and severity of many chronic complications associated directly with DM. ${ }^{12)}$

Considering complications related to TCC and reconstructive surgery, we treated midfoot Charcot arthropathy with simple management using hard-soled shoes or simple braces rather than TCC. The purpose of this study is to report clinical and radiological outcomes of midfoot Charcot arthropathy treated by our protocol. This study has the following hypotheses: (1) for treatment of midfoot Charcot arthropathy, hard-soled shoes are sufficient, instead of TCC; (2) unlike hindfoot-ankle arthropathy, midfoot Charcot arthropathy is rarely progressive. Therefore, instead of surgery for arch restoration, conservative treatment is recommended. Minor surgery such as simple bumpectomy can be performed for selective cases.

\section{METHODS}

\section{Subjects}

The protocol of this study was reviewed and approved by the Institutional Review Board of Asan Medical Center (IRB No. 20170711). A retrospective review of medical records was performed on 44 patients (51 feet) diagnosed as having midfoot Charcot arthropathy between October 2006 and July 2014. Exclusion criteria were (1) Charcot arthropathy of the forefoot, hindfoot, and ankle, (2) combined active deep infection such as abscess or osteomyelitis at diagnosis, (3) less than two years of follow-up after diagnosis, and (4) modified Eichenholtz stage 3 at first visit.

Of the total 44 patients (51 feet) with midfoot Charcot arthropathy, ten patients (13 feet) were excluded. Two patients (two feet) died of chronic kidney disease and diabetic complications. Four patients (six feet) did not come for follow-up. Four patients (five feet) were excluded for concomitant deep infection with osteomyelitis at the time of diagnosis. Finally, 34 patients (38 feet) were selected as subjects of this retrospective study.

All medical records of the included patients were retrospectively reviewed regarding gender, age, duration of diabetes before the onset of Charcot arthropathy, followup period, diabetes type, body mass index (BMI), hemoglobin A1c (HbA1c) level, creatinine level, and fasting glucose level at the time of diagnosis of Charcot arthropathy.

\section{Management of Midfoot Charcot Arthropathy Conservative treatment}

After the diagnosis of midfoot Charcot arthropathy, immediate weight-bearing was started in a hard-soled shoe. The patient was instructed to wear the shoe both indoors and outdoors. The hard-soled shoe was used for about 3 months after diagnosis; then, we recommended readymade rigid outsole shoe. A hard-soled shoe limits bending of the foot and toes. TCC was not applied in all patients to obviate cast-related problems, such as muscle atrophy and wound. There was no restriction on daily living activities. All patients were followed up every 2 to 4 weeks for the first three months and at monthly intervals for the next three months. Each patient returned for regular evaluation of the foot every three to six months.

\section{Selective bumpectomy}

During follow-up of midfoot Charcot arthropathy, we conducted simple bumpectomy for patients who complained of plantar pain due to a bony plantar bump and for those who had ulcer wound at the site of bony plantar bump.

\section{Radiological Evaluation}

At each visit, we obtained weight-bearing simple radiographs (anteroposterior, lateral, and oblique radiographs) of the foot. Outcomes of interest included progression of arch collapse, progression of bony prominence, and changes in Charcot stage.

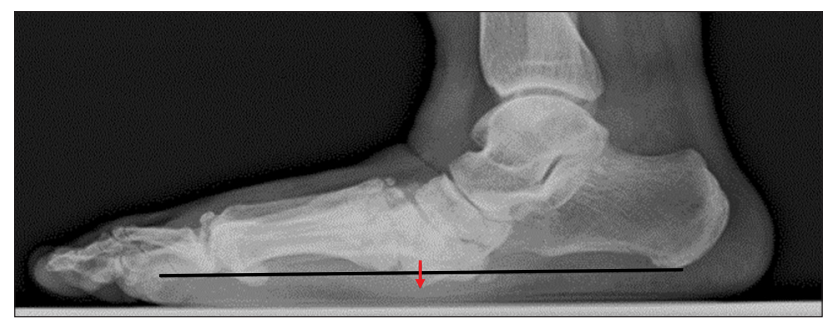

Fig. 1. With reference to the baseline (white line), the position change of the bottom bump in the initial and final foot lateral radiographs was assessed (arrow). 
Kim et al. Conservative Treatment of Midfoot Charcot Arthropathy

Clinics in Orthopedic Surgery • Vol. 11, No. 4, $2019 \bullet$ www.ecios.org

\section{Progression of arch collapse}

To assess the progression of arch collapse, we measured the talo-first metatarsal angle (Meary's angle) on weightbearing lateral radiographs of the foot at the time of diagnosis and at the last follow-up. Progression was classified according to the difference between the two measurements: (1) not flattened, when there was no change in Meary's angle; (2) mildly flattened, when the difference was less than $2^{\circ}$; (3) moderately flattened, when the difference was $2^{\circ}$ or more but less than $5^{\circ}$; and (4) severely flattened, when the difference was more than $5^{\circ}$.

\section{Progression of bony prominence (bump)}

To assess the progression of bony prominence, we checked the position of a bump on the bottom of the midfoot on the weight-bearing lateral radiograph at the time of diagnosis and at the last follow-up. On the weight-bearing lateral radiograph of the foot, a bottom line connecting the calcaneal medial tuberosity and the first metatarsal head was defined as a baseline (Fig. 1). The distance between this baseline and the bump on the bottom of the midfoot was then measured (arrow in Fig. 1). If the bump was located above the baseline, it was expressed as positive; if the bump was located below the baseline, it was expressed as negative. The position change of the bump in the initial and final lateral radiographs was assessed and classified as follows: (1) no progression, when there was no change; (2) mild progression, when the difference was less than $2 \mathrm{~mm}$; (3) moderate progression, when the difference was more than $2 \mathrm{~mm}$ and the bump at the final follow-up was above the bottom line; and (4) severe progression, when the difference was more than $2 \mathrm{~mm}$ and the bump a the final follow-up was below the bottom line.

\section{Changes in Eichenholtz stage}

The stage at presentation was classified according to the modified Eichenholtz stage ${ }^{13)}$ : (1) stage 0, radiographs without abnormal findings, but loss of protective sensation with swelling and erythema; (2) stage 1, radiographic evidence of fragmentation or dissolution; (3) stage 2, radiographs showing coalescence of fragments; and (4) stage 3 , radiographs showing reconstruction of fragments. We checked changes in stages between the first visit and the last follow-up. Three independent foot ankle specialists (YKK, SGS, SHP) evaluated the stage and the average values were used for analysis.

\section{Clinical Outcome Measures}

Ulcer occurrence, amputation, and bony bump-related plantar pain were examined. In addition, laboratory find- ings such as HbA1c level, serum creatinine level, and fasting glucose level were also assessed.

\section{Ulcer occurrence}

Ulcer occurrence was defined as the presence of plantar ulcer in the midfoot due to bony bumps of the involved extremity during follow-up. We excluded cases with diabetic foot ulcers not associated with bony bumps.

\section{Amputation}

Amputation of the involved foot due to progression of infection related to midfoot Charcot arthropathy was investigated.

\section{Bump-related plantar pain}

Patients' complaints of plantar pain around the bony bump were investigated.

\section{Statistical Analysis}

Student $t$-test was used to determine the difference in Meary's angle and bump position between initial and final follow-up. The duration of DM, age, BMI, HbAlc, serum creatinine level, and fasting glucose level were compared using Student $t$-test. Categorical parameters such as gender and diabetes type were analyzed by using Pearson chisquare test. A $p$-value less than 0.05 was considered statistically significant.

\section{RESULTS}

\section{Subjects}

Of 34 patients, 14 were men and 20 were women. Their mean age was 59 years (range, 26 to 75 years). The average follow-up was 32.9 months (range, 24 to 78 months). The average duration of diabetes before the onset of Charcot arthropathy was 20.7 years (range, 8 to 31 years). Seven patients had type $1 \mathrm{DM}$ and 27 patients had type $2 \mathrm{DM}$. Mean BMI of these patients was $25.6 \mathrm{~kg} / \mathrm{m}^{2}$ (range, 18.2 to $35.9 \mathrm{~kg} / \mathrm{m}^{2}$ ) at the time of diagnosis. Six patients had postrenal transplant. Nine patients had nephropathy.

\section{Radiological Evaluation}

\section{Progression of arch collapse}

Of 38 feet, eight (20\%) were not flattened, 12 (32\%) were mildly flattened, 14 (37\%) were moderately flattened, and four (10\%) were severely flattened. The difference between initial and final Meary's angles was not statistically significant in the not flattened, mildly flattened, and moderately flattened feet (all $p>0.05$ ). However, in the severely flattened feet, the difference was statistically significant $(p<$ 
Kim et al. Conservative Treatment of Midfoot Charcot Arthropathy

Clinics in Orthopedic Surgery • Vol. 11, No. 4, $2019 \bullet$ www.ecios.org

\section{Table 1. Radiographic Outcomes of Conservative Management of Midfoot Charcot Arthropathy}

\begin{tabular}{|c|c|c|c|c|}
\hline Variable & & Radiologic change & & $p$-value \\
\hline Arch flattening $\left({ }^{\circ}\right)$ & Initial Meary's angle & Final Meary's angle & Difference & \\
\hline No $(n=8,20 \%)$ & 3.5 & 3.5 & 0 & $>0.05$ \\
\hline Mild (n=12, 32\%) & 4.5 & 5.5 & 1.0 & $>0.05$ \\
\hline Moderate $(n=14,37 \%)$ & 5.2 & 8.5 & 3.3 & $>0.05$ \\
\hline Severe $(n=4,10 \%)$ & 3.3 & 19.5 & 16.2 & $<0.05$ \\
\hline Bump progression (mm) & Initial bump position & Final bump position & Migration & \\
\hline No $(n=8,20 \%)$ & 5.5 & 5.5 & 0 & $>0.05$ \\
\hline Mild $(n=14,37 \%)$ & 5.6 & 4.2 & 1.4 & $>0.05$ \\
\hline Moderate $(n=12,32 \%)$ & 4.7 & 1.2 & 3.5 & $<0.05$ \\
\hline Severe $(n=4,10 \%)$ & 2.3 & -5.2 & 7.5 & $<0.05$ \\
\hline
\end{tabular}

Table 2. Comparison of Demographic Characteristics between No or Mildly Flattened Feet and Moderately or Severely Flattened Feet

\begin{tabular}{|c|c|c|c|c|}
\hline Variable & Total & No or mild flattening & Moderate or severe flattening & $p$-value \\
\hline Number & 38 & 20 & 18 & \\
\hline Sex & & & & 0.701 \\
\hline Male & 14 & 8 & 6 & \\
\hline Female & 24 & 12 & 12 & \\
\hline Age (yr) & $59.1 \pm 9.3$ & $59.0 \pm 11.3$ & $59.2 \pm 6.9$ & 0.890 \\
\hline Duration of diabetes (yr) & $20.7 \pm 8.6$ & $18.3 \pm 7.1$ & $23.5 \pm 9.5$ & 0.012 \\
\hline Duration of follow-up (mo) & $32.9 \pm 8.9$ & $33.2 \pm 8.7$ & $32.5 \pm 8.9$ & 0.751 \\
\hline Body mass index $\left(\mathrm{kg} / \mathrm{m}^{2}\right)$ & $25.6 \pm 4.2$ & $25.5 \pm 4.3$ & $25.7 \pm 3.9$ & 0.692 \\
\hline Diabetes type & & & & 0.584 \\
\hline Type 1 & 10 & 6 & 4 & \\
\hline Type 2 & 28 & 14 & 14 & \\
\hline \multicolumn{5}{|l|}{ Lab finding } \\
\hline Hemoglobin A1c (\%) & $7.8 \pm 2.1$ & $7.6 \pm 2.1$ & $8.0 \pm 2.1$ & 0.382 \\
\hline Creatinine (mg/dL) & $3.5 \pm 4.0$ & $3.2 \pm 3.7$ & $3.9 \pm 2.5$ & 0.030 \\
\hline Fasting glucose level (mg/dL) & $158.2 \pm 24.2$ & $156.2 \pm 20.3$ & $160.3 \pm 25.2$ & 0.690 \\
\hline
\end{tabular}

Values are presented as mean \pm standard deviation.

0.05) (Table 1).

Factors contributing to progression of arch collapse were analyzed by comparing demographic characteristics between no or mildly flattening feet and moderately or severely flattened feet (Table 2). Duration of diabetes and creatinine level were significantly higher in the moderately or severely flattened feet. However, other factors such as gender, age, diabetes type, initial stage of Charcot arthropathy, BMI, HbA1c, or fasting glucose level were not significantly different between the two groups. 
Kim et al. Conservative Treatment of Midfoot Charcot Arthropathy

Clinics in Orthopedic Surgery • Vol. 11, No. 4, $2019 \bullet$ www.ecios.org

\section{Progression of bony prominence (bump)}

We classified the progression of bony prominence into four stages. Of 38 feet, eight (20\%) had no progression, 14 (37\%) had mild progression, 12 (32\%) had moderate progression, and four $(10 \%)$ had severe progression. Four patients with severe arch collapse and the four patients with severe bump progression coincided. The extent of progression was not statistically significant in no progression and mild progression feet $(p>0.05)$, whereas in moderate and severe progression feet, it was statistically significant $(p<$ 0.05) (Table 1).

\section{Changes in Eichenholtz stage}

The median value assessed by three independent observers at first visit was $1.13 \pm 0.20$ (range, 0 to 2). Of 38 feet, one foot was stage 0,31 feet were stage 1 , and six feet were stage 2 . The median value at the final follow-up was $2.71 \pm$ 0.25 . Of 38 feet, 11 feet were stage 2 and 27 feet were stage 3.

\section{Clinical Outcome Measures}

\section{Ulcer occurrence}

Foot ulcers related to bony bumps of Charcot arthropathy were found in two patients (two of 38 feet, 5.3\%). Of the two patients with a plantar ulcer lesion, one underwent below-knee amputation while the other underwent bumpectomy and wound repair. Plantar ulcer did not recur for 15 months after bumpectomy.

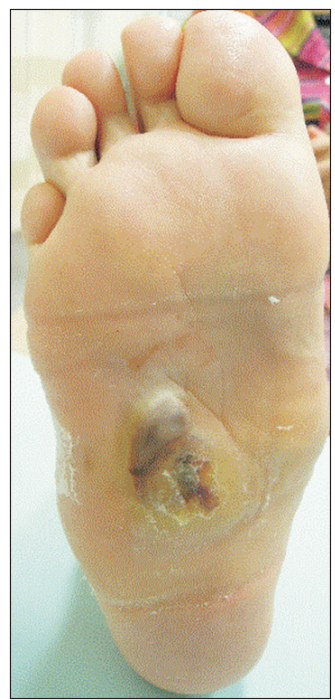

Fig. 2. A gross photo of a bump-related skin ulcer lesion on the plantar surface in a patient who underwent below-knee amputation.

\section{Amputation}

One patient (one of 38 feet, 2.6\%) underwent belowknee amputation. The patient was diagnosed as having plantar ulcer 20 months after the diagnosis of Charcot arthropathy. During the follow-up, the bump progressed and bump-related plantar ulceration developed (Fig. 2). Although we recommended simple bumpectomy, the patient refused and instead underwent flap surgery without bumpectomy at another hospital. However, the flap wound was necrotic and infection progressed; therefore, belowknee amputation was required.

\section{Bump-related plantar pain}

Three patients (three of 38 feet, 7.8\%) had bump-related plantar pain. One patient refused bumpectomy. The other two patients had no postoperative plantar pain after bumpectomy.

\section{DISCUSSION}

The goal of treatment in Charcot arthropathy is to achieve a stable, plantigrade foot without infection and ulcer to allow for ambulation in the long term. For this purpose, we use hard-soled shoes and selective bumpectomy, instead of major reconstructive surgery or TCC, in our patients with Charcot arthropathy. We believe that long-term immobilization after reconstructive surgery or inconvenience due to TCC will adversely affect the patient's functional ability.

Pinzur ${ }^{14)}$ reported that among 66 patients with midfoot Charcot arthropathy nonoperatively treated at a large tertiary center, a small group of patients remained unstable and unbraceable. The amputation rate was $9 \%$ in the study, which suggests that better techniques for treatment are needed. ${ }^{15)}$ In our study, midfoot Charcot arthropathy showed little progression with conservative treatment. Although arch collapse over $5^{\circ}$ and bump progression below the bottom line occurred in four out of 38 feet, all patients had plantigrade feet and functional ambulation. Although the bump progresses occasionally, when a bump-related problem arises, whether it is pain or a ulcer lesion, simple bumpectomy can prevent further complications. Only one of 38 feet underwent below-knee amputation because bumpectomy was not performed at a proper time and uncontrolled deep infection occurred without appropriate ulcer management. Thus, plantar ulcers over bony bumps should be treated early by debridement of necrotic tissue and bumpectomy to offload the affected area.

In this study, we suggested a simple treatment strategy that focuses on ulcer control rather than reconstruction surgery for midfoot Charcot arthropathy. Even in the 
Kim et al. Conservative Treatment of Midfoot Charcot Arthropathy

Clinics in Orthopedic Surgery • Vol. 11, No. 4, $2019 \bullet$ www.ecios.org

case with severe arch collapse, the conservative treatment was effective. And in the present study, one patient with severe arch collapse underwent simultaneous kidney-pancreas transplant after being diagnosed as having Charcot arthropathy. One patient was diagnosed as having Charcot arthropathy at 25 months after kidney transplant. Another patient with severe arch collapse was diagnosed as having Charcot arthropathy before kidney transplant. However, arch collapse rapidly progressed within 1 year after kidney transplant. Seo et al. ${ }^{16)}$ have reported that diabetic Charcot arthropathy may develop after successful pancreas transplant and concluded that attention should be paid to diabetic foot due to its high incidence at the initial stage of transplant. Our results are consistent with results of their study. Therefore, the surgeon needs to be cautious when performing transplant in patients with Charcot arthropathy. On the basis of the comparison of the degree of arch collapse between the minimally collapsed feet and significantly collapsed feet, we confirmed that the serum creatinine level and duration of diabetes are significant associated factors. The longer the diabetic period, the worse the kidney function and the lower the serum creatinine level. Poor kidney function can affect the metabolism of bones and the progression of Charcot arthropathy.

Studies on results of surgical treatment of Charcot midfoot arthropathy have demonstrated variable results depending on the bone quality, bone loss, vasculopathy, and the presence of infection with or without soft-tissue loss. Sammarco et al. ${ }^{17)}$ reported results of 22 patients who underwent surgical reconstruction for midfoot Charcot arthropathy. Three of 22 patients (13.7\%) had recurrent plantar ulcer that required additional surgery and eight patients (36\%) had hardware failure. Another study on 15 patients who underwent reconstructive surgery for midfoot Charcot arthropathy reported that one patient without prior ulceration developed deep infection that resulted in amputation. ${ }^{18)}$ Four feet $(26.7 \%)$ had nonunion, one of which was symptomatic and revision was required to obtain union. Open correction with internal fixation for Charcot arthropathy has been associated with a high rate of complications and failure due to infection, bone softening, resorption, fragmentation, and breakage of the implant. However, some studies have reported favorable results after surgical treatment. One study on 14 patients with Stage I midfoot Charcot arthropathy has reported successful healing of primary corrective arthrodesis without immediate or long-term complications or any recurrent ulceration during a mean follow-up period of 41 months. ${ }^{19)}$

TCC is most commonly used to treat neuropathic complications of Charcot arthropathy and neuropathic ulceration. Its effect has been reported in numerous studies. ${ }^{20)}$ However, successful TCC even in the hands of an experienced physician may result in subsequent ulcerations in $30 \%$ of patients during treatment. ${ }^{9)}$ It can also cause muscle wasting. Although we allowed full weight-bearing ambulation without TCC immediately after diagnosis of Charcot arthropathy, there were not many cases of arch collapse. Functional ambulation was also possible in all patients except for those who had medical comorbidities. Such good results might be because we prevented muscle wasting without applying TCC. The strengths of this study are that all patients had Charcot arthropathy due to DM and were treated with conservative treatment. However, this study also has some limitations. First, the study is retrospective in nature and based on a relatively short followup of a limited number of subjects. In addition, the study has no control group consisting of patients with the same condition but treated differently. Therefore, we had to compare our results with those of other previous reports. Second, although we tried to ensure accurate assessment of the Eichenholtz stage by using the mean values obtained among three independent foot ankle specialists, the interobserver reliability was not assessed by intraclass correlation coefficients. Despite these limitations, we found that arch collapse occurred only few cases of midfoot Charcot arthropathy even when comfortable ambulation in a hardsoled shoe was allowed at the beginning of the diagnosis. This was also resolved with simple bumpectomy. Tibiatalus-calcaneus arthrodesis surgery or rigid cast is necessary for hindfoot Charcot arthropathy However, midfoot Charcot arthropathy should be treated differently from hindfoot Charcot arthropathy: comfortable ambulation should be allowed to prevent ambulatory activity decline and muscle strength weakness.

In our study, we found that midfoot Charcot arthropathy is rarely progressive, unlike hindfoot-ankle arthropathy. Therefore, conservative treatment without restriction of ambulation is recommended. TCC is not mandatory in managing midfoot Charcot arthropathy. For some cases, simple bumpectomy with close follow-up might be needed to prevent catastrophic infection.

\section{CONFLICT OF INTEREST}

No potential conflict of interest relevant to this article was reported. 
Kim et al. Conservative Treatment of Midfoot Charcot Arthropathy

Clinics in Orthopedic Surgery • Vol. 11, No. 4, $2019 \bullet$ www.ecios.org

\section{REFERENCES}

1. Nather A, Bee CS, Huak CY, et al. Epidemiology of diabetic foot problems and predictive factors for limb loss. J Diabetes Complications. 2008;22(2):77-82.

2. Prieto-Perez L, Perez-Tanoira R, Petkova-Saiz E, et al. Osteomyelitis: a descriptive study. Clin Orthop Surg. 2014;6(1):205.

3. ElAlfy B, Ali AM, Fawzy SI. Ilizarov external fixator versus retrograde intramedullary nailing for ankle joint arthrodesis in diabetic Charcot neuroarthropathy. J Foot Ankle Surg. 2017;56(2):309-13.

4. Frykberg RG, Sage RA, Wukich DK, Pinzur MS, Schuberth JM. Charcot arthropathy. Foot Ankle Spec. 2012;5(4):26271.

5. Sanders LJ, Frykberg RG. Diabetic neuropathic osteoarthropathy: the Charcot foot. In: Frykberg RG, ed. The high risk foot in diabetes mellitus. New York: Churchill Livingstone; 1991. 297-338.

6. Silvampatti S, Nagaraja HS, Rajasekaran S. Midfoot Charcot arthropathy: overview and surgical management. J Foot Ankle Surg (Asia Pac). 2016;3(2):97-106.

7. Wukich DK, Raspovic KM, Hobizal KB, Sadoskas D. Surgical management of Charcot neuroarthropathy of the ankle and hindfoot in patients with diabetes. Diabetes Metab Res Rev. 2016;32 Suppl 1:292-6.

8. Lee DJ, Schaffer J, Chen T, Oh I. Internal versus external fixation of Charcot midfoot deformity realignment. Orthopedics. 2016;39(4):e595-601.

9. Guyton GP. An analysis of iatrogenic complications from the total contact cast. Foot Ankle Int. 2005;26(11):903-7.

10. El Oraby HA, Abdelsalam MM, Eid YM, El Hilaly R, Marzouk HA. Bone mineral density in type 2 diabetes patients with Charcot arthropathy. Curr Diabetes Rev. 2019;15(5):395401.

11. Papanas N, Maltezos E. Etiology, pathophysiology and clas- sifications of the diabetic Charcot foot. Diabet Foot Ankle. $2013 ; 4$.

12. Han HS, Kang SB. Relations between long-term glycemic control and postoperative wound and infectious complications after total knee arthroplasty in type 2 diabetics. Clin Orthop Surg. 2013;5(2):118-23.

13. Shibata T, Tada K, Hashizume C. The results of arthrodesis of the ankle for leprotic neuroarthropathy. J Bone Joint Surg Am. 1990;72(5):749-56.

14. Pinzur MS. Benchmark analysis of diabetic patients with neuropathic (Charcot) foot deformity. Foot Ankle Int. 1999;20(9):564-7.

15. Saltzman CL, Hagy ML, Zimmerman B, Estin M, Cooper R. How effective is intensive nonoperative initial treatment of patients with diabetes and Charcot arthropathy of the feet? Clin Orthop Relat Res. 2005;(435):185-90.

16. Seo DK, Lee HS, Park J, Ryu CH, Han DJ, Seo SG. Diabetic foot complications despite successful pancreas transplantation. Foot Ankle Int. 2017;38(6):656-61.

17. Sammarco VJ, Sammarco GJ, Walker EW Jr, Guiao RP. Midtarsal arthrodesis in the treatment of Charcot midfoot arthropathy. J Bone Joint Surg Am. 2009;91(1):80-91.

18. Assal M, Stern R. Realignment and extended fusion with use of a medial column screw for midfoot deformities secondary to diabetic neuropathy. J Bone Joint Surg Am. 2009;91(4):812-20.

19. Simon SR, Tejwani SG, Wilson DL, Santner TJ, Denniston NL. Arthrodesis as an early alternative to nonoperative management of Charcot arthropathy of the diabetic foot. J Bone Joint Surg Am. 2000;82(7):939-50.

20. Armstrong DG, Nguyen HC, Lavery LA, van Schie CH, Boulton AJ, Harkless LB. Off-loading the diabetic foot wound: a randomized clinical trial. Diabetes Care. 2001;24(6):101922. 